\title{
The impact of L2 proficiency on cross-language influence during L2 word-processing
}

\section{Francesca M. Branzi ${ }^{1,2}$, Ya-Ning Chang ${ }^{1,3}$, Claudia Gaele ${ }^{1}$, Dora Alexopoulou ${ }^{1}$ \\ 1. University of Cambridge; 2. University of Liverpool; 3. National Cheng Kung University.}

\section{Introduction}

We investigated the relationship between $\mathrm{L} 2$ proficiency and the language control strategies employed during $L 2$ word-processing to cope with cross-language interference. Our main hypothesis is that proactive inhibition of the non-target language (L1) is the best cognitive strategy to optimise L2 performance when L1 and L2 lexical/phonological representations do not overlap. This strategy should be especially implemented by $L 2$ high proficient individuals. In fact, if links between L2 (English) and the conceptual system are strong (high proficiency), these individuals may not need to pass from L2 (English) to L1 (Chinese) to activate semantic information. To this end, we tested a group of native speakers of Chinese (L1) with various levels of proficiency in English (a second language acquired later in life - i.e., L2) in a task that required to decide whether English words presented in pairs were related in meaning or not. Crucially, L2 learners were unaware of the fact that half of the words concealed a character repetition when translated into Chinese. This allowed to measure the 'character repetition effect' without a character repetition when translated into Chinese. The $\mathrm{CR}$ effect reflects the extent to which L1 phonology is engaged during L2 processing. According to our main hypothesis that high proficient L2 learners would apply L1 inhibition, we predicted to observe a negative correlation between the magnitude of the $C R$ effect and L2 proficiency measures.

A second hypothesis is that high proficient $L 2$ learners should be more accurate when accessing the meaning of L2 words as compared to less proficient L2 learners. This should result in at least two predicted outcomes: first, a positive correlation between between L2 proficiency and the magnitude of the 'semantic relatedness effect' (SR), obtained from the comparison between semantically-related versus semantically-unrelated word pairs. (CR), which is obtained by comparing word-pairs with versus overall task accuracy and L2 proficiency; second, a correlation

\section{Results}

\section{BEST and self-rated L2 proficiency}

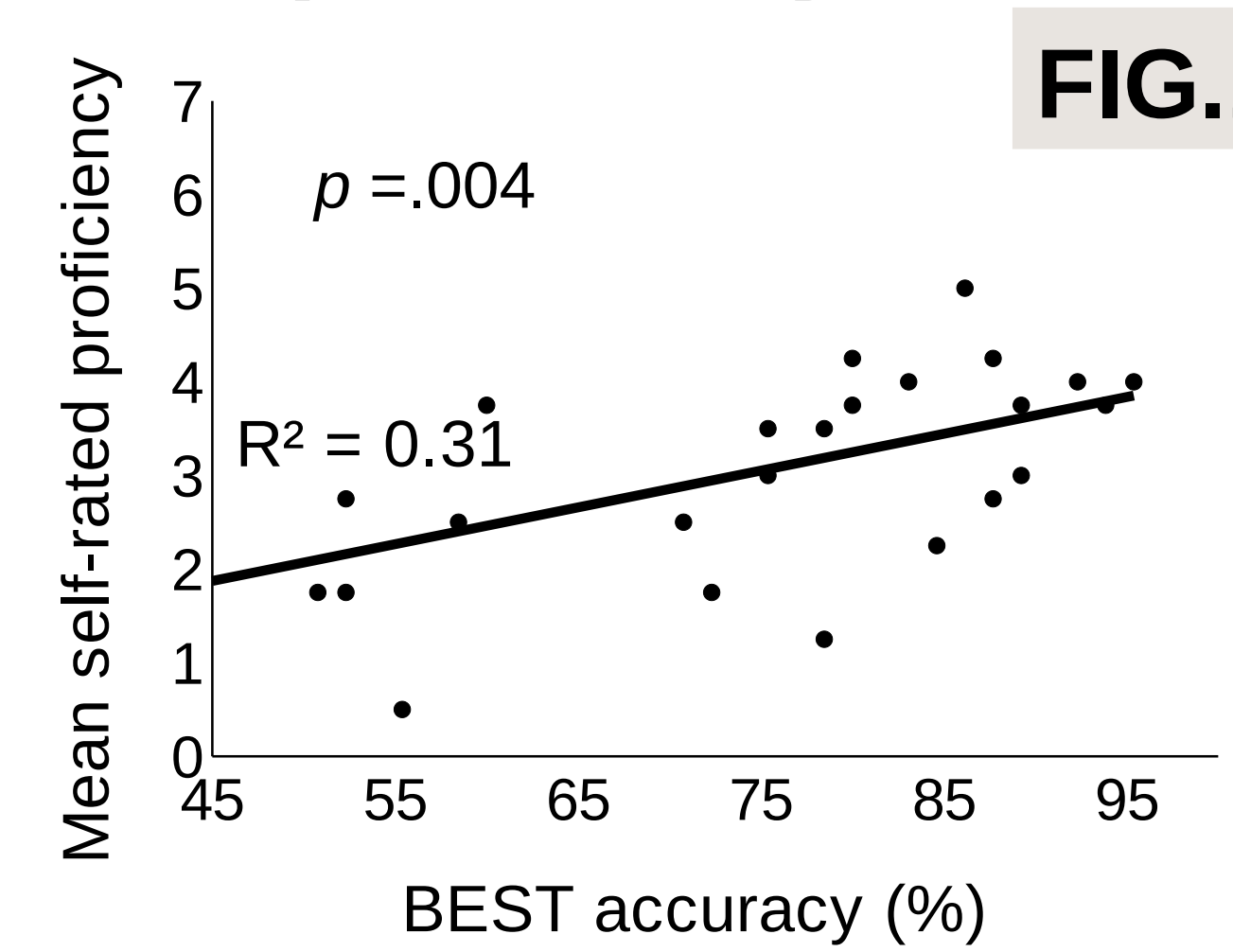

L2 proficiency and overall
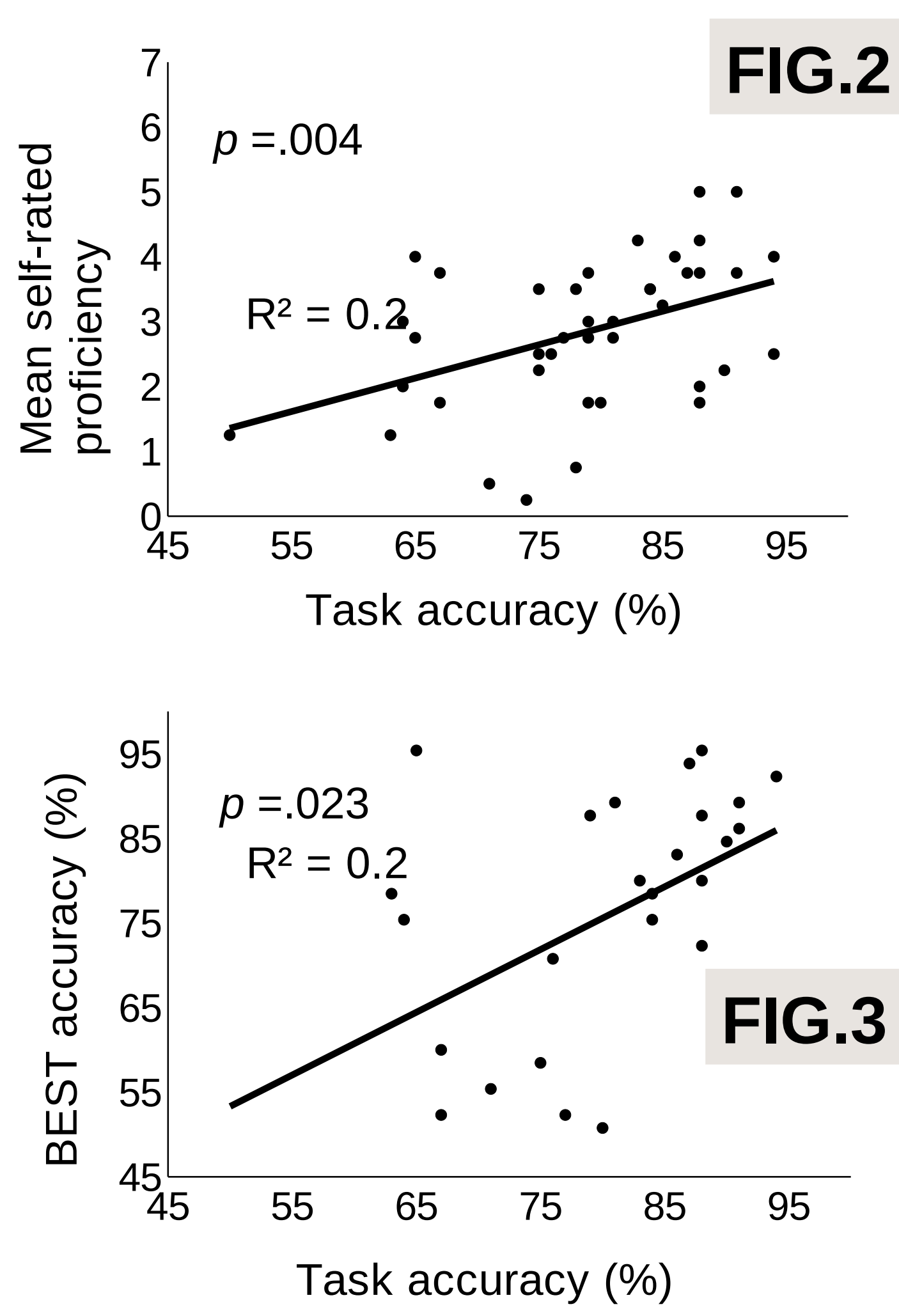

Figure 1. Self-rated and BEST measures of L2 proficiency are significantly correlated. Figure 2 3. L2 proficiency significantly predicts overall task accuracy (semantically related trials). task accuracy

\section{Methods}

Participants. 40 healthy and right-handed native speakers of Chinese (18 females, mean age $=$ $21.4 \pm 2.72$ years), with various levels of proficiency in English (L2) (mean age of L2 acquisition = $9.3 \pm 2.8$ years)

Measures of $L 2$ proficiency. We collected different measures of L2 proficiency. First, we collected self-rated L2 proficiency (from $0=$ none to very high $=7$ ) regarding (i) writing, (ii) reading, (iii) speaking and (iv) oral comprehension. Furthermore, we collected objective measures of L2 proficiency with the BEST test (De Bruin et al., 2017).

Stimuli. From Thierry and Wu (2007). Experimental trials: 100 semantically-related English word pairs with $(C R+, n=50)$ and without a character repetition $(C R-, n=50)$ divided in 4 blocks; Filler trials (not analysed): 40 semantically-unrelated word pairs also divided in 4 blocks. Half trials with character repetition (CR+), the rest without character repetition (CR-).

Task. As in Thierry and Wu (2007), the task required to indicate if probe ('Post') and target ('Mail') were semantically related (two-alternative-forced-choice task). The experiment was written in JavaScript, using the jsPsych library (de Leeuw, 2015; https://www.jspsych.org/) and set up using JATOS (Lange et al., 2015; http://www.jatos.org/).
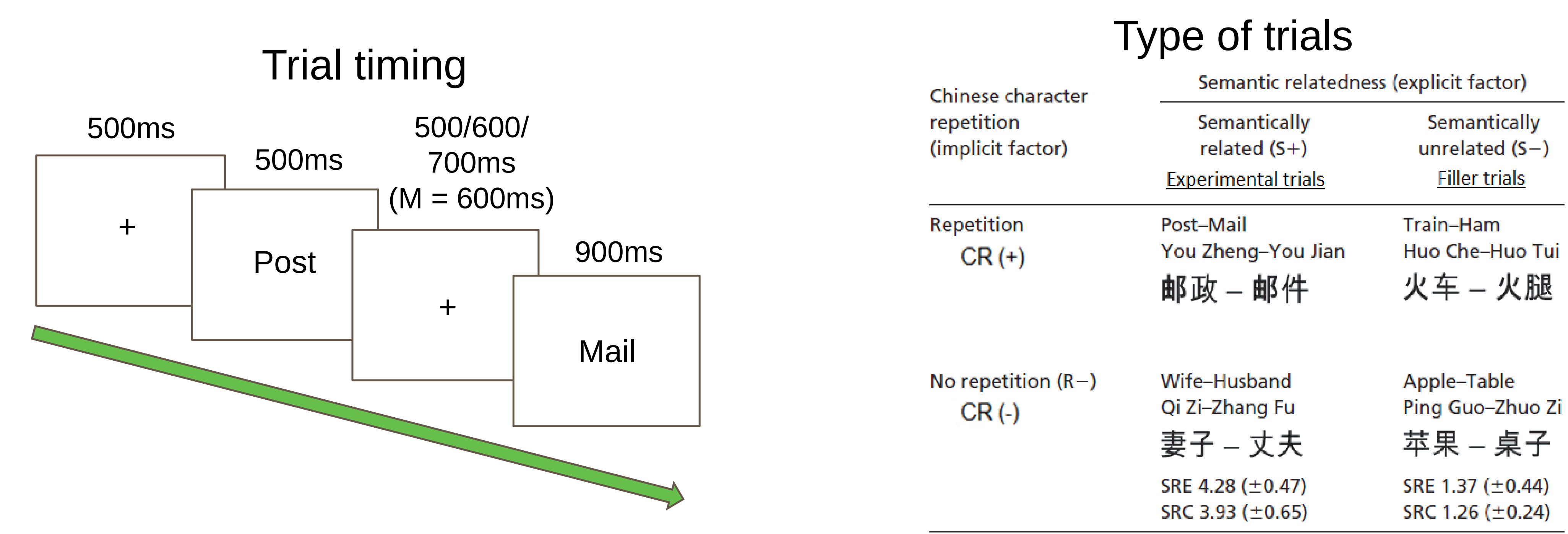

\section{Discussion}

Our results do not support our main hypothesis that inhibitory control is applied on the non-target language (L1), especially for high proficient L2 learners. Rather, we found that L2 proficiency is positively (but not negatively) correlated with the magnitude of the CR effect (see Fig. 10 and 11).
Francesca.Branzi@liverpool.ac.uk

\section{Semantic Relatedness effect ('SR effect')}
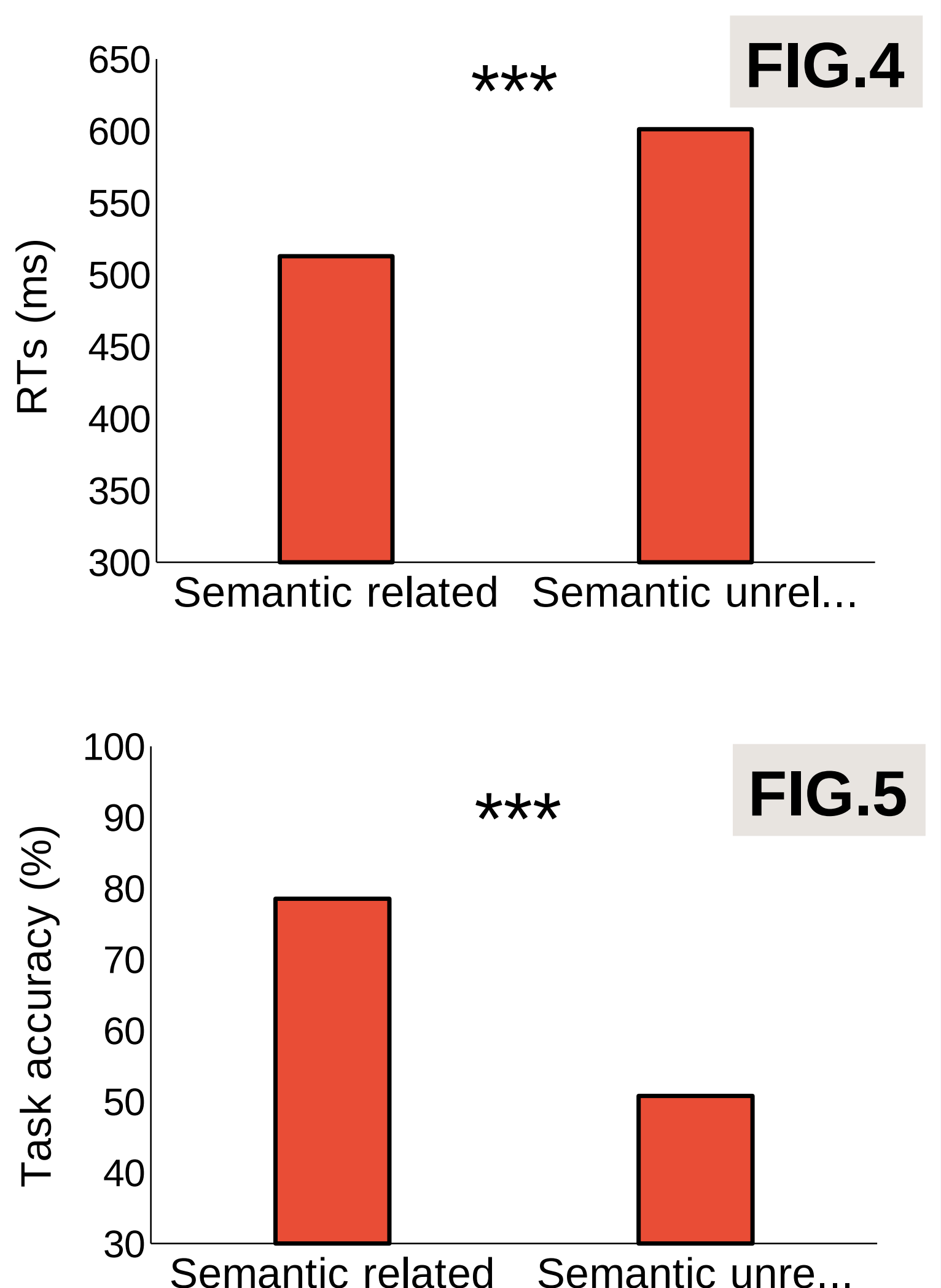

Figure 4 and 5 . According to Thierry and Wu (2007), the task is easier when probe and target are semantically related versus when probe and target are semantically unrelated.

\section{Character Repetition effect ('CR effect')}
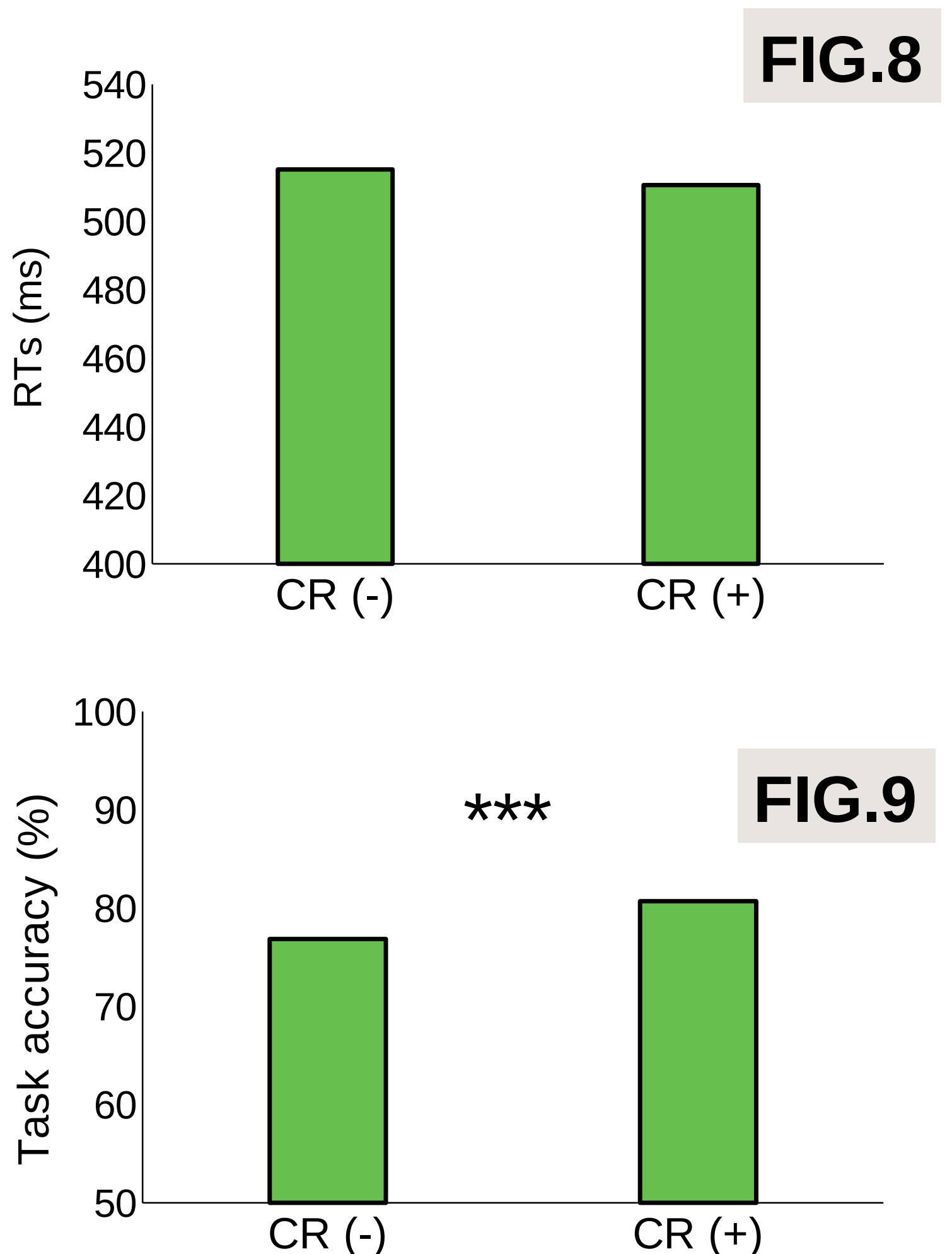

Figure 8 and 9 . The $\mathrm{CR}$ effect in (accuracy) suggests that the task is easier when L1 translation of probe and target share some phonological representations.
SR effect and $L 2$ proficiency
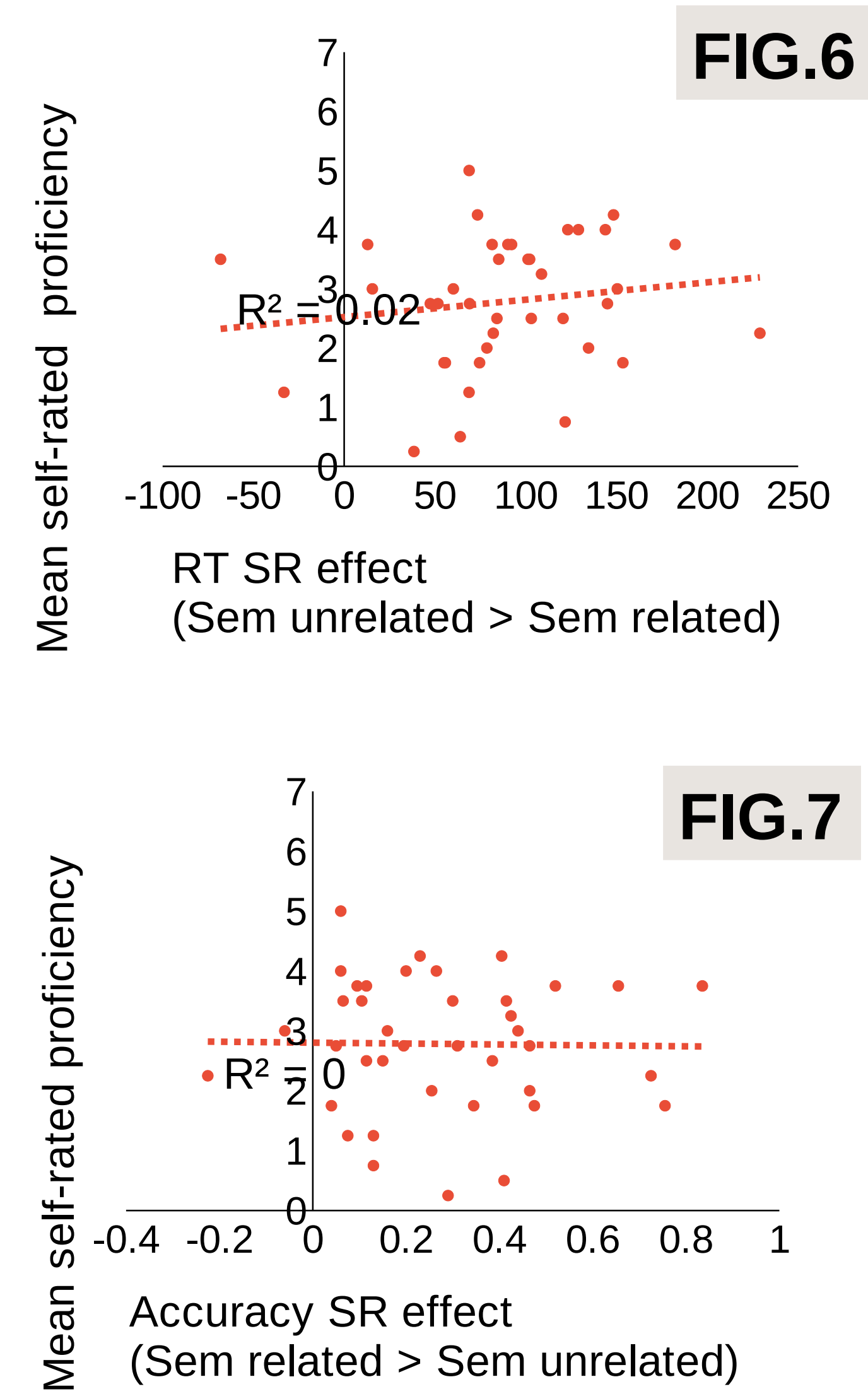

Figure 6 and 7 . The SR effect is not predicted by L2 proficiency measures.

\section{$C R$ effect and $L 2$ proficiency}

\section{FIG.10}

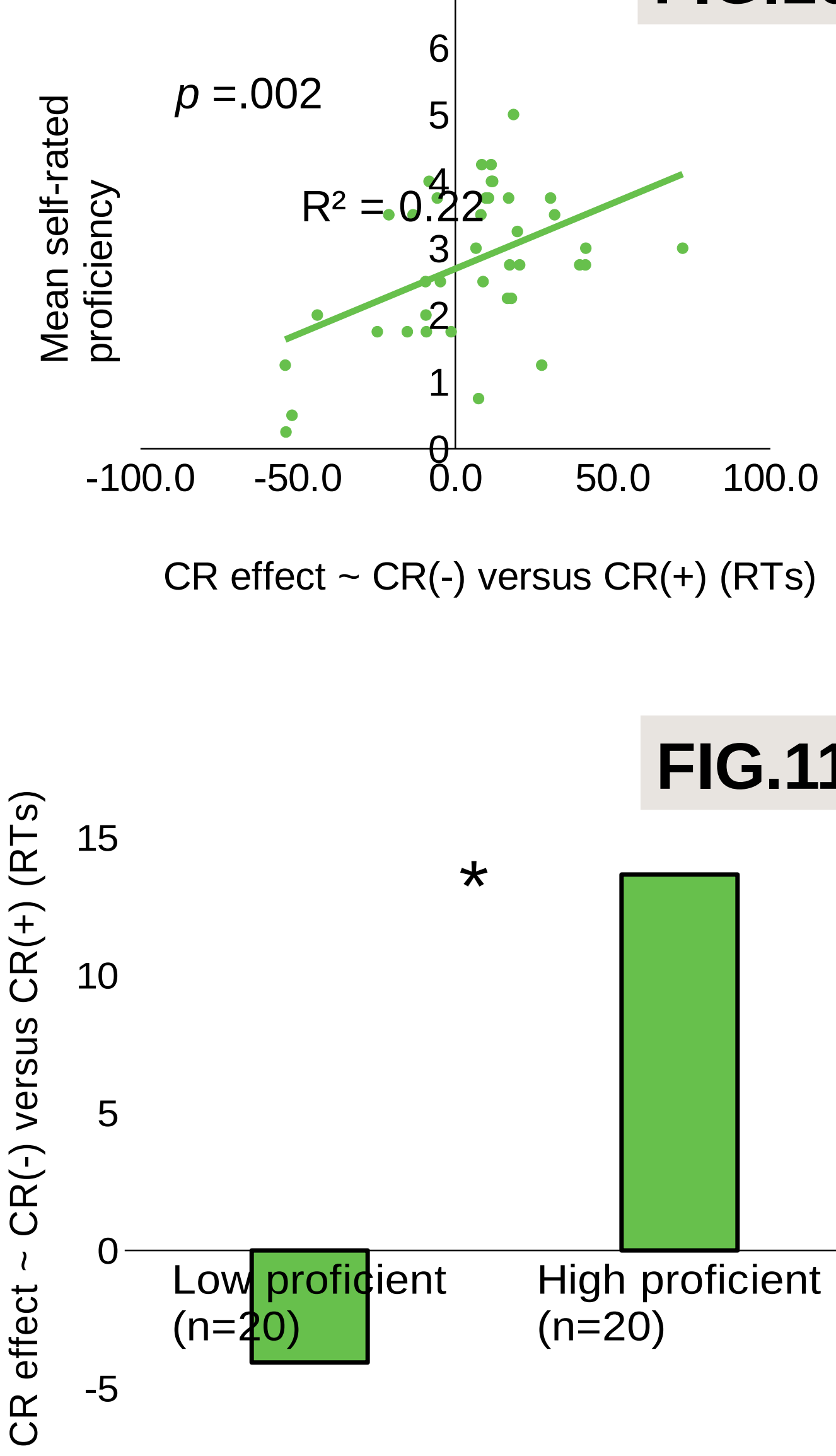

Figure 10 and 11. The magnitude of the CR effect is predicted by L2 self-rated proficiency measures. A significant CR effect is observed only in L2 high proficient learners.

These results can be explained at least in two ways:

(1) High and low proficient L2 learners use qualitatively different mechanisms for language control (low proficient use inhibition versus high proficient use language selection/activation - see Costa et al., 2004). (2) Links between the L2 lexicon and the semantic system are stronger in high versus low proficient bilinguals. Thus, in high proficient L2 learners, the activation from the semantic system would spread more strongly in the bilingual lexicon, towards phonology of the non-target language-L1. Finally, the significant SR effect confirms our second hypothesis that high proficient L2 learners are more accurate when accessing the meaning of L2 words as compared to less proficient individuals (see Figures 2-3).

References: Thierry and Wu, PNAS (2007); De Bruin et al., (2017) Frontiers in psychology. Lange et al., PloS one (2015); De Leeuw, Behavior research methods (2015); Costa et al., Journal of memory and Language (2004). 